\title{
How oral infections can influence chronic kidney disease - a review of the literature
}

\author{
Carla Cruvinel Pontes, Usuf ME Chikte \\ Division of Health Systems and Public Health, Department of Global Health, Stellenbosch University, Cape Town, South Africa.
}

\section{ABSTRACT}

A healthy mouth is necessary for optimal health and quality of life. However, oral health is often compromised in adults with chronic kidney disease (CKD). The aim of this review is to present the scientific foundations behind the connection between oral diseases and chronic kidney disease in adults, discuss common oral conditions and their systemic effects, investigate biological pathways through which oral infections affect the body, and provide guidelines for physicians/nephrologists. Prevalence of oral disease is increased in CKD, including periodontal disease, oral mucosal lesions, edentulousness, xerostomia, gingival overgrowth in immunosuppressed patients and potentially caries. There is moderate to strong evidence to support a negative impact of oral infections in CKD, particularly periodontal disease, with systemic inflammation, bacteraemia of oral origin, endothelial function and gut dysbiosis being potential pathways for this interaction. Poor oral health can be a hidden source of infection and has been associated with increased mortality in CKD patients. Elimination of potential foci for oral infections is crucial before renal transplantation. Frequent dental monitoring is essential for these patients and should be part of a multidisciplinary approach to manage CKD, with special attention to end-stage kidney disease.

Keywords: oral infection; oral disease and CKD; periodontitis and CKD.

\section{INTRODUCTION}

A healthy mouth is necessary for optimal health and quality of life [I]. However, oral health is often compromised by highly prevalent diseases such as caries, periodontal disease, mucosal lesions and occasionally oral cancer. Globally, over 3.5 billion people have oral disease. It is the most common non-communicable disease (NCD) worldwide, which constitutes a public health challenge, affecting most individuals throughout their lives [2]. Poor oral health is linked to a multitude of problems, which are not limited to the destruction of local tissues, pain, discomfort and impaired oral function, but it also affects the systemic environment, with the potential to increase inflammation in other body organs and systems [3].

Periodontal disease is a group of conditions that affect the tissues surrounding the teeth, with gingivitis and periodontitis being the most common [4]. Elevated levels of pro-inflammatory cytokines and acute phase response mediators have been reported in the serum, plasma and gingival tissues of patients with periodontitis. A multitude of studies provide evidence on a potential relationship between periodontitis and general conditions, including chronic kidney disease (CKD), cardiovascular disease, diabetes, respiratory disease, rheumatoid arthritis, cancer, cognitive impairment and pregnancy outcomes [5].

Increased prevalence and severity of oral diseases have been reported in patients with CKD [6,7]. Considering that infection and inflammation originating from the oral cavity have the potential to contribute to systemic inflammation, it is imperative to understand the impact of oral health on CKD and why it needs to be integrated into the overall management of CKD.

The aim of this review of the literature is to present the scientific foundations of the connection between oral 
diseases and CKD in adults, to discuss the most common oral conditions in CKD and their effect on the systemic environment, to investigate the biological pathways through which oral infections affect the body and to provide guidelines for physicians and nephrologists who treat patients with CKD.

\section{COMMON ORAL CONDITIONS IN PATIENTS WITH CKD}

\section{Periodontal disease}

Periodontal disease is a term that includes disorders affecting the periodontium, that is, the tissues that surround the teeth, and are typically characterized by immuneinflammatory response to bacterial pathogens in the oral biofilm [8]. Dental plaque or biofilm constitutes a reservoir of microorganisms, with over 500 microbial species that colonize different oral surfaces, including the epithelium, dental surfaces and the space below the gingival margin, known as gingival sulcus (in health) or pocket (in periodontal disease) [9].

The most prevalent diseases affecting the periodontium are gingivitis and periodontitis. Whereas gingivitis represents initial inflammation limited to gingival soft tissues, periodontitis represents the progression of gingivitis, where the inflammatory process reaches deeper layers of connective and osseous tissues, leading to destruction of supporting structures that hold the teeth in place [10]. Periodontal disease can be challenging to identify by non-dental professionals due to the typically discrete nature of signs and symptoms.

Gingivitis can present the following signs and symptoms due to gingival inflammation [ $1 \mathrm{l}]$ :

a. Erythema, swelling of gingival tissues

b. Soreness

c. Gingival bleeding induced by eating, toothbrushing or flossing

d. Bad breath, metallic or altered taste

e. Difficulty eating.

According to severity and complexity of management, periodontitis is classified as stage I (initial), stage II (moderate), stage III (severe with risk for tooth loss) or stage IV (severe with risk of loss of the dentition). It is also reported in terms of grades to describe progression as slow (grade A), moderate (grade B) and rapid (grade C) [12].

The signs and symptoms of periodontitis include all those described for gingivitis and a few additional ones [10]:

a. Presence of calculus

b. Receding gums; teeth can look longer c. Loose or separating teeth

d. Change in the bite; change in the fit of partial dentures

e. Pus between gingiva and teeth

f. Pain when chewing.

Management of periodontal disease relies on controlling the biofilm and relevant modifiable risk factors. Scaling and root planning, also known as debridement, combined with effective oral hygiene procedures is the usual initial approach for periodontal treatment. If the mechanical treatment is not able to arrest disease progression, surgical and/or regenerative procedures can be performed, followed by regular professional maintenance. Systemic or local antibiotics are sometimes employed during periodontal treatment. For the majority of systemically healthy individuals, efficient daily oral hygiene habits are successful at limiting periodontal disease [2].

\section{Periodontal disease in CKD patients}

Several studies have reported an association between periodontal disease and CKD. Fisher et al. found that periodontitis was independently associated with CKD and suggested a potential bidirectional link that can be related to diabetes and hypertension [13]. Duran and Erdemir reported that in patients undergoing haemodialysis, there was a correlation between worse periodontal conditions and duration of dialysis [14]. A link between elevated serum antibodies to periodontal pathogens and impaired kidney function was reported in a study by Kashirsagar et al.; the association remained significant after control for traditional risk factors [15]. The Third National Health and Nutrition Examination Survey (NHANES III) for the US population included 86 I participants with CKD (6\%). The I0-year allcause mortality rate in those participants increased from $32 \%$ to $41 \%$ when periodontitis was present, characterizing a strong association between periodontitis and mortality in stage 3-5 CKD patients [16]. Periodontitis has been linked to reduced serum albumin in patients on haemodialysis therapy in two different studies, which can be a risk factor for increased mortality $[17,18]$.

The association between periodontitis and CKD has been extensively explored in systematic reviews [19-23]. The first such review and meta-analysis on periodontitis and CKD was published in 2013 by Chambrone et al. Their findings indicate an increased risk for CKD in the presence of periodontitis and a potential beneficial effect of periodontal treatment on glomerular filtration rate (eGFR) [19]. In another systematic review, Deschamp-Lenhardt and co-workers observed that most of the studies included reported increased incidence of periodontitis in CKD patients. These authors performed a meta-analysis in which a strong association between severe periodontitis and 
CKD was present $(O R=2.39[1.70-3.36])$ even after adjusting for significant risk factors for CKD [20].

In a systematic review from 2018, Kapellas et al. [22] found that CKD patients had higher odds of having periodontitis $(O R=1.69[0.84-3.40])$. In that same year, a different systematic review by Zhao et al. presented results from 38 studies on the association of periodontitis and CKD, including directional and non-directional associations [2 I]. The majority of studies (75\%) indicated a significant nondirectional relationship between CKD and periodontitis, which was supported by the 2.12 odds ratio resulting from the meta-analysis. These authors pointed out that, despite the 5 stages of CKD, over half of the studies focused on patients with end-stage kidney disease (ESKD) [2I].

In 2020, the same group evaluated the effect of periodontal treatment in CKD patients in one randomized controlled clinical trial (RCT) and four case series, with most studies including dialysis patients. The authors found insufficient evidence for the beneficial effect of non-surgical periodontal treatment in patients with CKD, as evaluated in terms of estimated glomerular filtration rate (eGFR) and serum creatinine and pointed out the need for more RCTs [23]. In contrast, based on results from five RCTs, Yue et al. reported a significant moderate reduction of highly sensitive CRP (hsCRP) after non-surgical periodontal treatment in haemodialysis and peritoneal dialysis patients [24].

Interestingly, malnutrition is a frequent problem faced by dialysis patients and data from various studies support a link between periodontitis and markers of malnutrition in dialysis and haemodialysis patients $[25,26]$.

In conclusion, the current literature supports a moderate to strong association between periodontitis and CKD, while the potential benefits of periodontal treatment on the systemic inflammation in end-stage kidney disease require further investigation, potentially due to the limited number of interventional studies. The potential link between malnutrition and periodontitis in CKD patients requires further investigation.

\section{Caries}

Caries is the most common NCD globally, leading to destruction of dental tissues because of exposure of dental surfaces to acids produced by oral bacteria in the presence of carbohydrates. Untreated caries can cause pain and discomfort associated with cavities, pulpitis (inflammation of the dental nerve) and ultimately tooth loss [27].

Currently, there is a lack of consensus on the relationship between caries and CKD, with some investigations reporting no differences between healthy people and CKD patients [28,29], others reporting a higher prevalence of caries [30], and some reporting lower caries prevalence in CKD patients [3I].

In a systematic review and meta-analysis by Limeira et al., caries prevalence in adults with CKD and in healthy controls was compared; these authors reported lower caries scores in CKD patients [32]. Similar findings of lower prevalence of caries were reported for children and adolescents with CKD in a systematic review by Andrade et al. [33]. The higher salivary urea concentration in CKD patients can possibly explain the higher capacity for remineralization of dental surfaces and lower prevalence of caries [34].

\section{Edentulousness}

The number of teeth present has a direct influence on masticatory function, with the presence of occluding premolar and molar teeth being a requirement for adequate mastication. The main causes of tooth loss are periodontitis and caries, which have been linked to several conditions due to inappropriate diet [35]. One prospective study reported a link between edentulousness and increased mortality in haemodialysis patients over a 12-month period [36]. Similarly, Ruokonen et al. reported fewer teeth present in deceased haemodialysis patients, suggesting a potential link between tooth loss and CKD [37].

\section{Oral mucosal alterations}

Oral mucosal lesions are more prevalent in cases of severe CKD and these patients are particularly prone to fungal infections. In a large study including over 4000 adults on haemodialysis, $40 \%$ of the sample had at least one oral mucosal lesion [38]. In a systematic review on oral lesions in CKD patients, oral candidiasis was present in approximately $20 \%$ of adults with stages $1-5$, and dialysis and oral herpetic lesions were reported in $2.0-2.9 \%$ of dialysis patients [39]. Abnormal lip pigmentation has been reported in $48 \%$ of patients with end-stage kidney disease in whom an association between oral lesions and GFR was reported [40].

Uncommon alterations in the oral mucosa of uraemic patients include oral ulcers, petechiae, bruises and uraemic stomatitis [4I]. The last is a specific condition seen in renal patients that is due to mucosal irritation by ammonia or its compounds. It is typically characterized by white plaques on the buccal mucosa, on the floor of the mouth and tongue, with pain, bad taste and a burning sensation being common symptoms. These lesions tend to improve after dialysis and observing oral hygiene with the use of antiseptic mouthwashes [42]. In kidney transplant patients, oral hairy leukoplakia and lichenoid lesions are common in the tongue, particularly in patients undergoing cyclosporine therapy, presenting as painless white patches with an irregular surface [4I]. 


\section{Xerostomia}

Xerostomia, also known as dry mouth, and reduced salivary flow have been reported in 28-59\% of ESRD patients, with polyuria, medication and alterations in the salivary glands being reported as the most common causes [40]. Saliva plays a crucial role in the maintenance of oral health. The reason why xerostomia decreases patients' quality of life is the associated symptoms of dysphagia, dysgeusia, discomfort and increases the risk of infections, caries and periodontitis. Typical signs of xerostomia include glossitis, peeling lips, pale oral mucosa and cervical caries [39].

Management of xerostomia includes mechanical stimulation of salivary glands (such as by chewing gum), use of saliva substitutes or medication (pilocarpine, angiotensinconverting-enzyme inhibitors alone or combined with angiotensin-receptor blockers). Several medications can cause xerostomia, including antihypertensive agents, psychotropic medication, anticholinergic drugs and sympathetic agonists [6].

\section{Other oral conditions}

Gingival overgrowth can be a side effect of immunosuppressive cyclosporine therapy in renal transplant patients or of calcium channel blockers in dialysis patients, which might require surgical excision of gingival tissues to re-establish gingival morphology and improve oral hygiene. Up to $70 \%$ of patients on cyclosporine can develop gingival overgrowth, whereas the prevalence in patients taking calcium channel blockers has been reported as 10-20\% [43].

Halitosis is mainly caused by volatile sulphur compounds (VSCs). In CKD, elevated blood and salivary urea levels have been associated with bad breath. Around half of dialysis patients present with halitosis; dimethyl sulphide and methyl mercaptan have been reported as the most prevalent VSCS in CKD-related halitosis. Dialysis can improve CKD-related halitosis, as it helps reduce blood urea nitrogen levels [44].

Higher calculus formation has been reported in patients with CKD, which has been linked to altered urea concentrations, leading to lower salivary $\mathrm{pH}$ and increased precipitation of calcium and phosphorus, thereby increasing calculus formation $[36,46]$.

In line with the increased prevalence of oral conditions in CKD patients, a recent systematic review investigated oral health-related quality of life (OHRQoL) in patients with end-stage kidney disease; it reported reduced OHRQoL for haemodialysis and renal transplantation patients, sug-

\section{EVIDENCE ON HOW ORAL INFECTIONS CAN CONTRIBUTE TO SYSTEMIC INFLAMMATION}

Chronic diseases, including CKD, share common risk factors with periodontitis, including exposure to tobacco, alcohol consumption, diabetes, increasing age and poor diet with high sugar content [47,48]. In a study by Fisher et al., periodontitis was considered a non-traditional risk factor for CKD given the association between the two diseases, which remained significant after adjustment for traditional risk factors [49]. The mechanisms behind the link between periodontitis and systemic conditions include systemic inflammation as a result of local inflammation in the periodontal tissues, bacteraemia, endothelial dysfunction and potential oral and gut dysbiosis [50].

\section{Systemic inflammation}

In periodontitis, elevated production of inflammatory mediators such as IL- I $\beta$, TNF- $\alpha$ and IL-6 have been reported in periodontal tissues, saliva and gingival crevicular fluid [51,52]. Increased levels of these markers have also been reported in serum, which can lead to raised CRP levels and potential damage to the kidneys, suggesting that locally produced cytokines can reach the circulation and contribute to systemic inflammation [53]. In a recent study by Rapone et al., periodontal status were associated with CRP serum levels in haemodialysis patients [54].

loannidou and co-workers evaluated the effect of periodontitis on CRP levels of patients with CKD through the NHANES III data set. In total, $42 \%$ of periodontitis, $27 \%$ of periodontally healthy patients and $53 \%$ of edentulous CKD patients had serum CRP above $0.3 \mathrm{mg} / \mathrm{dL}$, suggesting a strong association between periodontitis and serum CRP levels in CKD [55]. Additional studies support the finding of a mild acute-phase systemic inflammatory response resulting from periodontitis, as reported in a systematic review from Paraskevas et al. [56]. Periodontal treatment has been shown to moderately reduce hs-CRP in haemodialysis and dialysis patients [24], although not all studies are able to report reduced serum inflammatory markers or renal function markers after periodontal treatment [23].

Anaemia is a prevalent finding in CKD patients, and despite treatment with erythropoiesis stimulatory agents (ESA), some patients fail to improve haemoglobin levels [57]. Interestingly, periodontitis has been associated with inflammation-related anaemia in a recent study involving over 800 patients. Periodontitis was associated with reduced haemoglobin, mean corpuscular haemoglobin $(\mathrm{MCH})$ and $\mathrm{MCH}$ concentration. In this study, 14-15.7\% of periodontitis patients presented anaemia according to the $\mathrm{WHO}$ criteria, whereas $8.4-10.2 \%$ of healthy controls were 
anaemic $[58,59]$. One of the mechanisms behind anaemia and haemoglobin variability in CKD patients treated with ESA is cytokine-induced inflammation, which is a shared characteristic of CKD and periodontitis. Thus, in anaemic CKD patients, oral infections, particularly periodontitis, can be a hidden source of inflammation and unresponsive haemoglobin levels in ESA-treated patients.

\section{Bacteraemia}

Another potential explanatory pathway for the influence of periodontitis on CKD is the entrance of bacteria or bacterial products, such as lipopolysaccharides, through ulcerated periodontal tissues into the bloodstream, with the potential to cause damage to the kidney endothelium [15].

Several lines of evidence support the role of bacteraemia of oral origin in systemic conditions. Certain periodontal pathogens, such as Aggregatibacter actinomycetemcomitans and Porphyromonas gingivalis, have the ability to invade macrophages and/or dendritic cells and escape the host response, which could be important in the context of bacteraemia [60]. Furthermore, the DNA and RNA of periodontitis bacteria have been identified in atheromatous lesions [61,62]. Levels of circulating bacteria have been reported to be higher in systemically healthy patients with periodontitis than in gingivitis patients [63]. While the immune system of a healthy person is expected to be able to eliminate circulating bacteria, bloodstream infections lead to higher mortality in CKD patients, particularly endstage kidney disease [64]. Collectively, these data suggest that bacteraemia can be relevant to the connection between systemic inflammation in periodontitis and CKD.

Of note, infective endocarditis (IE), which can lead to renal involvement in up to $60 \%$ of IE cases and potentially to ESRD, can be caused by oral bacteria $[65,66]$. The most common oral bacteria linked to IE are streptococci from the viridans group. Dental treatment can potentially cause bacteraemia, although the risk is significantly higher for toothbrushing [67]. Although toothbrushing and flossing can increase the risk of short-term bacteraemia, good oral hygiene reduces the risk for IE in the long term [68]. In the presence of periodontal disease, the ulcerated gingival sulcus is believed to facilitate the entrance of oral streptococci bacteria into the systemic circulation [66]. Good oral hygiene and elimination of infection in the oral cavity can reduce the bacterial load and help prevent IE from oral bacteria.

\section{Endothelial dysfunction}

The high risk of cardiovascular disease in CKD patients cannot be totally explained by traditional risk factors such as diabetes and hypertension. In CKD, endothelial dys- function increases the risk of cardiovascular disease [69]. Periodontitis has been associated with endothelial dysfunction in healthy patients with no cardiovascular risk factors and in patients with hypertension. In the presence of periodontitis, forearm blood flow responses to acetylcholine were significantly reduced compared to subjects without periodontitis [70]. These findings have been confirmed by another study, in which flow-mediated dilatation of the brachial artery was positively associated with periodontitis [7I]. Interestingly, periodontal treatment has been associated with reduced serum CRP and IL-6 and improved acetylcholine-induced vasodilation in systemically healthy patients and in patients with hypertension [70], which was confirmed by a meta-analysis reporting a beneficial effect of periodontal treatment on endothelial function [72]. Hence, endothelial dysfunction could be a biological link through which periodontitis contributes to worsening of CKD, increasing the risk for cardiovascular disease.

\section{Gut dysbiosis}

Gut dysbiosis has been suggested as a novel risk factor for the progression and development of complications in CKD due its potential to increase systemic inflammation and oxidative stress. One of the proposed biological pathways for gut dysbiosis in CKD is the disruption of the intestinal epithelium and the translocation of uraemic toxins from the gastrointestinal lumen to the circulation [73].

Periodontitis has also been linked to gut dysbiosis resulting from ingestion of dysbiotic oral bacteria through swallowed saliva. Studies suggest that periodontal pathogens can reach the gut and change the local microbiota, increase gut permeability and contribute to endotoxemia and systemic inflammation [74]. Indeed, P. gingivalis has shown resistance to stomach acids, being able to reach the colon and alter its function [75,76]. Under healthy conditions, oral bacteria have limited ability to colonize the intestine; however, in the presence of certain systemic diseases, such as colon cancer and inflammatory bowel syndrome and HIV infection, oral bacteria have been reported in elevated amounts in the gut [77].

Given that gut dysbiosis has been linked to malnutrition [78], and periodontitis has also been associated with markers of malnutrition $[25,26,79]$, there is biological plausibility in the link between oral diseases, nutrition, gut health and general health.

Lourenço et al. [80] investigated the gut microbiome in periodontitis patients and reported less diversity in the intestinal microbiome in periodontitis cases compared to healthy controls. Furthermore, several oral bacteria asso- 
ciated with periodontal inflammation were identified in the gut microbiome of these individuals irrespective of their periodontal status [80]. An in vitro study on the periodontal pathogens Porphyromonas gingivalis and Prevotella intermedia suggested that frequent swallowing of dead bacteria can affect pathogens in the intestines, resulting in increased cytotoxicity and upregulation of virulence genes [8I]. Thus, despite the limited number of human studies on gut dysbiosis in periodontitis patients and on the potential mechanisms involved, this is a new area of research which can represent a novel pathway for oral bacteria to contribute to systemic inflammation in CKD and other systemic diseases [74].

\section{THE IMPORTANCE OF PREVENTION AND TREATMENT OF ORAL CONDITIONS FOR PATIENTS WITH CKD}

Disease in the oral cavity can impair quality of life, affecting important functions such as mastication, digestion and speech [82]. The consequences of oral conditions go beyond the oral cavity, potentially contributing to increased inflammation in the body, as discussed in the previous sections. Individuals with CKD are more susceptible to infections, the reason why untreated oral conditions, which tend to be more prevalent and severe than in the general population, can become a source of infection and adversely affecting the disease. Dental and medical management of patients with CKD can be challenging for several reasons, including their increased tendency for bleeding, the additional risk of infections and interactions between medications [83]. Frequent dental monitoring is crucial for these patients and should be part of a multidisciplinary approach to managing CKD and ESKD.

In Table I we present a summary of the oral lesions that are frequently observed in patients with impaired kidney function and the proposed dental and medical approach to address these conditions (adapted from Abed et al. [84]).

\section{Oral health and mortality in CKD patients}

Untreated dental conditions and poor oral health can contribute to premature death in patients with end-stage kidney disease, as reported in a prospective study on 4205 haemodialysis patients. The mortality rate was associated with edentulism and caries, whereas good oral hygiene practices were associated with increased survival [36]. These findings were confirmed by another study, in which more oral infections and fewer teeth were present in deceased pre-dialysis patients [37].

The relation between periodontitis and mortality due to cardiovascular disease in haemodialysis patients was investigated by Kashirsagar et al. [85]. Moderate to severe periodontitis was positively associated with cardiovascular

Table I. Summary of oral lesions in relation to impairment of renal function and the proposed integrated management approach.

\begin{tabular}{|c|c|c|}
\hline Renal function & Oral conditions & Suggested integrated approach \\
\hline \multirow[b]{2}{*}{ Removal of metabolic waste } & $\begin{array}{l}\text { Halitosis - "ammonia breath" can develop due to } \\
\text { high urea levels combined with poor oral hygiene and } \\
\text { stomatitis. }\end{array}$ & $\begin{array}{l}\text { Control of uraemic toxins, improvement in oral hygiene } \\
\text { habits. }\end{array}$ \\
\hline & $\begin{array}{l}\text { Uraemic stomatitis - white/grey membranes in the oral } \\
\text { mucosa; can be combined with ulcers, typically painful. }\end{array}$ & $\begin{array}{l}\text { Benzydamine hydrochloride }(0.15 \%) \text { can be prescribed } \\
\text { to alleviate uraemic stomatitis symptoms. When } \\
\text { urea levels return to normal, the stomatitis heals } \\
\text { spontaneously after approximately } 3 \text { weeks. }\end{array}$ \\
\hline \multirow[t]{2}{*}{ Water and electrolyte balance } & $\begin{array}{l}\text { Xerostomia (dry mouth) - saliva flow can decrease } \\
\text { and salivary glands can become swollen; linked to } \\
\text { changes in protein and electrolytes. }\end{array}$ & $\begin{array}{l}\text { Several palliative measures can help patients with } \\
\text { xerostomia to avoid development of caries and perio- } \\
\text { dontal disease. }\end{array}$ \\
\hline & $\begin{array}{l}\text { Dental calculus - can result from increased salivary pH } \\
\text { and altered mineral metabolism. }\end{array}$ & Frequent professional dental cleaning. \\
\hline \multirow[t]{3}{*}{ Blood pressure control } & $\begin{array}{l}\text { Oral lichenoid reaction - can be a side effect of beta- } \\
\text { blocker medications to control high blood pressure. }\end{array}$ & $\begin{array}{l}\text { No elective dental treatment if blood pressure is } \\
\text { uncontrolled; emergency dental treatment requires } \\
\text { liaison with physician. }\end{array}$ \\
\hline & $\begin{array}{l}\text { Gingival overgrowth - can develop in patients taking } \\
\text { cyclosporine and calcium channel blockers. }\end{array}$ & $\begin{array}{l}\text { Consider change in medication, gingival surgery can } \\
\text { be required to restore morphology and facilitate oral } \\
\text { hygiene. }\end{array}$ \\
\hline & $\begin{array}{l}\text { Oral candidiasis - patients can become more } \\
\text { prone to fungal infections in the mouth after renal } \\
\text { transplantation. }\end{array}$ & Topical antifungal therapy for 7-14 days. \\
\hline
\end{tabular}


mortality - an association that remained significant after adjusting for smoking, diabetes, hypertension, age and gender [85]. These findings were corroborated in the study by Sharma et al. [16].

The importance of oral health for peritoneal dialysis and haemodialysis

Owing to the higher prevalence of oral infection and other conditions in both pre-dialysis and dialysis patients, frequent dental follow-ups are essential to reduce the risk of local and systemic complications. Prevention and treatment of periodontitis needs to be a priority to decrease the inflammatory burden. The salivary rate of patients on dialysis and haemodialysis can be lower, with xerostomia being a frequent finding, which can lead to higher risk of oral diseases. It is important to maintain regular dental followups to manage xerostomia and avoid the consequent development of other oral conditions, such as caries and periodontitis. Salivary flow has been reported to improve after kidney transplantation [86]. Halitosis can be another oral condition in uraemic patients (uraemic breath) and usually improves after dialysis.

\section{The importance of oral health for kidney transplantation}

Oral infections need to be systematically eliminated before kidney transplantation and use of immunosuppressive medications. In general, kidney transplant patients have a relatively high prevalence of periodontitis and oral conditions. Furthermore, exposure to cyclosporine or calcium channel blockers can have side effects on the oral cavity, such as gingival overgrowth, leukoplakia, recurrent oral ulcers, viral infections, oral candidiasis and malignancies, particularly lip cancer $[87,88]$. Daily oral hygiene can become challenging in the presence of gingival overgrowth, which can increase the risk of periodontitis and caries [89].

Zwiech et al. reported increased risk of acute rejection 12 months after kidney transplantation in patients with poor oral health, suggesting the potential of poor oral health to adversely affect the function of transplanted kidneys [90]. Together with medical monitoring, kidney transplantation patients require frequent dental monitoring to allow for early detection and treatment of oral conditions to avoid local and systemic complications [9/].

Thus, all stages of CKD require frequent dental follow-ups, including preventive and restorative measures, particularly ESKD. However, palliative and preventive treatment should take place only 6 months after kidney transplanta136 tion, as this is a critical phase for graft rejection and the most intensive phase of immunosuppression. No invasive dental treatment should be performed before 6 months post kidney transplantation [84].

\section{KEY INFORMATION ABOUT ORAL INFECTIONS IN CKD PATIENTS FOR PHYSICIANS AND NEPHROLOGISTS}

- There is a relatively high prevalence of oral disease in CKD patients, including periodontitis, xerostomia, lower salivary flow, calculus, taste disturbances, halitosis (uraemic odour), oral mucosal lesions and potentially caries. Early detection and treatment can minimize the need for comprehensive dental care.

- Optimal oral hygiene measures are important to reduce the bacterial burden associated with the dental biofilm and prevent oral infections. Patient motivation to maintain adequate daily oral hygiene is critical.

- The identification of periodontal diseases can be challenging and requires specific examination with a periodontal probe, which can register gingival bleeding and the depth of the gingival pocket in millimetres, revealing the severity and extent of the disease. Radiographs can also help in the identification of alveolar bone loss, resulting from periodontitis. Periodontal treatment can potentially help reduce systemic markers of inflammation.

- Management of xerostomia includes use of saliva substitutes and sugar-free chewing gum to increase salivary flow, avoidance of mouth breathing, alcohol, tobacco, caffeine and alcohol-containing mouthwashes.

- Mastication can be poor as a result of periodontitis and edentulousness, leading to poor nutritional status, which is also frequently linked to CKD. Control of oral diseases to reduce risk of tooth loss and adequate tooth replacement should be considered to restore masticatory function in CKD patients.

- Diagnosis of oral mucosal lesions in CKD may require a biopsy to exclude potential malignancies.

- When preparing patients for kidney transplantation, it is crucial for them to undergo thorough screening and treatment for oral infections before starting on immunosuppressive medications and receiving kidney transplantation.

- An integrated approach to healthcare in CKD is necessary, with oral health being an essential part of the overall medical strategy. Upon referral, the nephrologist should inform the dentist about relevant aspects of the patient's medical history, the stage of the disease, current medications and the latest laboratory test results. 


\section{CONCLUSIONS}

CKD is often associated with poor oral health, increased prevalence of periodontitis, caries, edentulism, calculus, oral mucosal lesions, halitosis and xerostomia. Untreated oral conditions can become a source of infection, being detrimental to the systemic environment owing to the potential to worsen general inflammation, endothelial function, nutritional status, quality of life and mortality in CKD patients. Frequent monitoring and treatment of oral infections is crucial in CKD patients, particularly prior to renal transplantation. Maintenance of a healthy and functional dentition and periodontium should be an integral part of the general medical approach for CKD patients, as it can contribute to better nutrition, lower general inflammation and infection, improved health outcome and longer survival.

\section{REFERENCES}

I. Dye BA. The global burden of oral disease: research and public health significance. J Dent Res. 2017; 96:36I-363.

2. Jin L, Lamster I, Greenspan J, Pitts N, Scully C, Warnakulasuriya S. Global burden of oral diseases: emerging concepts, management and interplay with systemic health. Oral Dis. 2016; 22:609-619.

3. Cardoso EM, Reis C, Manzanares-Céspedes MC. Chronic periodontitis, inflammatory cytokines, and interrelationship with other chronic diseases. Postgrad Med. 2018; 130:98-104.

4. Petersen PE, Ogawa $\mathrm{H}$. The global burden of periodontal disease: towards integration with chronic disease prevention and control. Periodontol. 2000. 2012; 60:15-39.

5. Falcao A, Bullón P. A review of the influence of periodontal treatment in systemic diseases. Periodontol. 2000. 2019; 79:1 17-128.

6. Akar H, Akar GC, Carrero JJ, Stenvinkel P, Lindholm B. Systemic consequences of poor oral health in chronic kidney disease patients. Clin J Am Soc Nephrol. 201 I; 6:218-226.

7. Schubert C, Nadeem M, Davids MR, Stephen L. Association between periodontitis and systemic inflammation in patients with end-stage renal disease. S Afr Dent J. 2009; 64:470-473.

8. Armitage GC. Development of a classification system for periodontal diseases and conditions. Ann Periodontol. 1999; 4:1-6.

9. Vieira Colombo AP, Magalhães CB, Hartenbach FARR, Martins do Souto R, Maciel da Silva-Boghossian C. Periodontal-diseaseassociated biofilm: A reservoir for pathogens of medical importance. Microb Pathog. 2015; 94:27-34.

I0. Papapanou PN, Sanz M, Buduneli N, Dietrich T, Feres M, Fine DH, et al. Periodontitis: Consensus report of workgroup 2 of the 2017 World Workshop on the Classification of Periodontal and Peri-Implant Diseases and Conditions. J Periodontol. 2018; 89:S173-S182

II. Chapple ILC, Mealey BL, Van Dyke TE, Bartold PM, Dommisch H, Eickholz P, et al. Periodontal health and gingival diseases and conditions on an intact and a reduced periodontium: Consensus report of workgroup I of the 2017 World Workshop on the Classification of Periodontal and Peri-Implant Diseases and Conditions. J Periodontol. 2018; 89:S74-S84.

12. Caton JG, Armitage G, Berglundh T, Chapple ILC, Jepsen S, Kornman KS, et al. A new classification scheme for periodontal and peri-implant diseases and conditions - Introduction and key changes from the 1999 classification. J Periodontol. 2018
13. Fisher MA, Taylor GW, Shelton BJ, Jamerson KA, Rahman M, Ojo $\mathrm{AO}$, et al. Periodontal disease and other nontraditional risk factors for CKD. Am J Kidney Dis. 2008; 51:45-52.

14. Duran I, Erdemir EO. Periodontal treatment needs of patients with renal disease receiving haemodialysis. Int Dent J. 2004; 54:274-278.

15. Kshirsagar A V., Offenbacher S, Moss KL, Barros SP, Beck JD. Antibodies to periodontal organisms are associated with decreased kidney function: The dental atherosclerosis risk in communities study. Blood Purif. 2007; 25: |25-|32.

16. Sharma P, Dietrich T, Ferro C], Cockwell P, Chapple ILC. Association between periodontitis and mortality in stages 3-5 chronic kidney disease: NHANES III and linked mortality study. J Clin Periodontol. 2016; 43:104-| I3.

17. Kshirsagar A V., Craig RG, Beck JD, Moss K, Offenbacher S, Kotanko $P$, et al. Severe periodontitis is associated with low serum albumin among patients on maintenance hemodialysis therapy. Clin J Am Soc Nephrol. 2007; 2:239-244.

18. Rodrigues VP, Libério SA, Lopes FF, Thomaz EBFA, Guerra RNM, Gomes-Filho IS, et al. Periodontal status and serum biomarkers levels in haemodialysis patients. J Clin Periodontol. 20|4; 4|:862-868.

19. Chambrone L, Foz AM, Guglielmetti MR, Pannuti CM, Artese HPC, Feres $M$, et al. Periodontitis and chronic kidney disease: a systematic review of the association of diseases and the effect of periodontal treatment on estimated glomerular filtration rate. J Clin Periodontol. 2013; 40:443-456.

20. Deschamps-Lenhardt S, Martin-Cabezas R, Hannedouche T, Huck $O$. Association between periodontitis and chronic kidney disease: Systematic review and meta-analysis. Oral Dis. 2019; 25:385-402.

21. Zhao D, Khawaja AT, Jin L, Li KY, Tonetti M, Pelekos G. The directional and non-directional associations of periodontitis with chronic kidney disease: A systematic review and meta-analysis of observational studies. J Periodont Res. 2018; 53:682-704.

22. Kapellas K, Singh A, Bertotti M, Nascimento GG, Jamieson LM. Periodontal and chronic kidney disease association: A systematic review and meta-analysis. Nephrology. 2019; 24:202-212.

23. Zhao D, Khawaja AT, Jin L, Chan KW, Tonetti M, Tang SCW, et al. Effect of non-surgical periodontal therapy on renal function in chronic kidney disease patients with periodontitis: a systematic review and meta-analysis of interventional studies. Clin Oral Investig. 2020; 24:1607-1618.

24. Yue H, Xu X, Liu Q, Li X, Xiao Y, Hu B. Effects of non-surgical periodontal therapy on systemic inflammation and metabolic markers in patients undergoing haemodialysis and/or peritoneal dialysis: A systematic review and meta-analysis. BMC Oral Health. 2020; 20: | 8. DOI: | 0.1 | 86/s | 2903-020- |004-I.

25. Cengiz MI, Bal S, Gökçay S, Cengiz K. Does periodontal disease reflect atherosclerosis in continuous ambulatory peritoneal dialysis patients? J Periodontol. 2007; 78:1926-1934.

26. Chen LP, Chiang CK, Chan CP, Hung KY, Huang CS. Does periodontitis reflect inflammation and malnutrition status in hemodialysis patients? Am J Kidney Dis. 2006; 47:8I 5-822.

27. Selwitz RH, Ismail Al, Pitts NB. Dental caries. Lancet. 2007; 9555:5।-59.

28. Tadakamadla J, Kumar S, Mamatha GP. Comparative evaluation of oral health status of chronic kidney disease (CKD) patients in various stages and healthy controls. Spec Care Dent. 20। 4; 34:122-126.

29. Bots CP, Poorterman JHG, Brand HS, Kalsbeek H, Van Amerongen $\mathrm{BM}$, Veerman $\mathrm{ECl}$, et al. The oral health status of dentate patients with chronic renal failure undergoing dialysis therapy. Oral Dis. 2006; 12:176-180.

30. Menezes CRSD, Pereira ALA, Ribeiro CCC, Chaves CO, Guerra RNM, Thomaz ÉBAF, et al. Is there association between chronic kidney disease and dental caries? A case-controlled study. Med Oral Patol Oral Cir Bucal. 2019; 24:e21I-e216. 
31. Davidovich E, Schwarz Z, Davidovitch M, Eidelman E, Bimstein E. Oral findings and periodontal status in children, adolescents and young adults suffering from renal failure. I Clin Periodontol. 2005; 32:1076-1082.

32. Limeira FIR, Yamauti M, Moreira AN, Galdino TM, de Magalhães CS, Abreu LG. Dental caries and developmental defects of enamel in individuals with chronic kidney disease: Systematic review and meta-analysis. Oral Dis. 2019; 25: I 446-| 464.

33. Andrade MRTC, Antunes LAA, Soares RMDA, Leão ATT, Maia LC, Primo LG. Lower dental caries prevalence associated to chronic kidney disease: A systematic review. Pediatr Nephrol. 20|4; 29:77|-778.

34. Anuradha BR, Katta S, Kode VS, Praveena C, Sathe N, Sandeep N, et al. Oral and salivary changes in patients with chronic kidney disease: A clinical and biochemical study. J Indian Soc Periodontol. 2015; 19: 297-30I.

35. Kailembo A, Preet R, Stewart Williams J. Common risk factors and edentulism in adults, aged 50 years and over, in China, Ghana, India and South Africa: Results from the WHO Study on global AGEing and adult health (SAGE). BMC Oral Health. 2016; 17. DOI:I0.1 I86/s I2903-016-0256-2.

36. Palmer SC, Ruospo M, Wong G, Craig JC, Petruzzi M, De Benedittis $M$, et al. Dental health and mortality in people with end-stage kidney disease treated with hemodialysis: A multinational cohort study. Am J Kidney Dis. 2015; 66:666-676.

37. Ruokonen H, Nylund K, Furuholm J, Meurman JH, Sorsa T, Kotaniemi K, et al. Oral health and mortality in patients with chronic kidney disease. J Periodontol. 2017; 88:26-33.

38. Ruospo M, Palmer SC, Graziano G, Natale P, Saglimbene V, Petruzzi $M$, et al. Oral mucosal lesions and risk of all-cause and cardiovascular mortality in people treated with long-term haemodialysis: The ORAL-D multinational cohort study. PLoS One. 2019; 14(6):e02 |8684. DOI: 10.137|/journal.pone.02 | 8684.

39. Ruospo M, Palmer SC, Craig JC, Gentile G, Johnson DW, Ford PJ, et al. Prevalence and severity of oral disease in adults with chronic kidney disease: A systematic review of observational studies. Nephrol Dial Transplant. 20 14; 29:364-375.

40. Oyetola EO, Owotade FJ, Agbelusi GA, Fatusi OA, Sanusi AA. Oral findings in chronic kidney disease: Implications for management in developing countries. BMC Oral Health. 2015; 15:24. DOI: I 0.1 I 86/s | 2903-015-0004-z.

41. Dioguardi M, Caloro GA, Troiano G, Giannatempo G, Laino L, Petruzzi M, et al. Oral manifestations in chronic uremia patients. Ren Fail. 2016; 38: 1-6.

42. Liao C, Wu C. Uremic stomatitis. QJM. 20 I7; I I0:247-248.

43. Ciavarella D, Guiglia R, Campisi G, Di Cosola M, Di Liberto C, Sabatucci $A$, et al. Update on gingival overgrowth by cyclosporine $A$ in renal transplants. Med Oral Patol Oral Cir Bucal. 2007; I2:el 9-25.

44. Honarmand M, Farhad-Mollashahi L, Nakhaee A, Sargolzaie F. Oral manifestation and salivary changes in renal patients undergoing hemodialysis. J Clin Exp Dent. 2017; 9:e207-e210.

45. Davidovich E, Davidovits M, Peretz B, Shapira J, Aframian DJ. The correlation between dental calculus and disturbed mineral metabolism in paediatric patients with chronic kidney disease. Nephrol Dial Transplant. 2009; 24:2439-2445.

46. Schmalz G, Patschan S, Patschan D, Ziebolz D. Oral health-related quality of life in adult patients with end-stage kidney diseases undergoing renal replacement therapy - A systematic review. BMC Nephrol. 2020; 21:I54. DOI: I0.I I 86/s I 2882-020-0I 824-7.

47. Albandar JM. Epidemiology and risk factors of periodontal diseases. Dent Clin North Am. 2005; 49:5 17-532.

48. Levey AS, Coresh J, Balk E, Kausz AT, Levin A, Steffes MW, et al. National Kidney Foundation practice guidelines for chronic kidney disease: Evaluation, classification, and stratification. Ann Intern Med. 2003; 139:137-147.
49. Fisher MA, Taylor GW, Shelton BJ, Jamerson KA, Rahman M, Ojo $\mathrm{AO}$, et al. Periodontal disease and other nontraditional risk factors for CKD. Am J Kidney Dis. 2008; 51:45-52.

50. Kramer CD, Genco CA. Microbiota, immune subversion, and chronic inflammation. Front Immunol. 20 I7; 8:255. DOI: 10.3389/ fimmu.2017.00255.

51. Zekeridou A, Mombelli A, Cancela J, Courvoisier D, Giannopoulou C. Systemic inflammatory burden and local inflammation in periodontitis: What is the link between inflammatory biomarkers in serum and gingival crevicular fluid? Clin Exp Dent Res. 2019; 5:128-135.

52. Almaghlouth AA, Cionca N, Cancela JA, Décaillet F, Courvoisier DS, Giannopoulou C, et al. Effect of periodontal treatment on peak serum levels of inflammatory markers. Clin Oral Investig. 20I4; |8:2113-2121.

53. Hayashi C, Gudino C V., Gibson FC, Genco CA. Pathogen-induced inflammation at sites distant from oral infection: Bacterial persistence and induction of cell-specific innate immune inflammatory pathways. Mol Oral Microbiol. 2010; 25:305-316.

54. Rapone B, Converti I, Santacroce L, Cesarano F, Vecchiet F, Cacchio $L$, et al. Impact of periodontal inflammation on nutrition and inflammation markers in hemodialysis patients. Antibiotics (Basel). 2019; 8:204. DOI: I 0.3390/antibiotics8040209.

55. Ioannidou E, Swede H, Dongari-Bagtzoglou A. Periodontitis predicts elevated C-reactive protein levels in chronic kidney disease. J Dent Res. 2011; 90:1411-1415.

56. Paraskevas S, Huizinga JD, Loos BG. A systematic review and meta-analyses on C-reactive protein in relation to periodontitis. J Clin Periodontol. 2008; 35:277-290.

57. Kanbay M, Perazella MA, Kasapoglu B, Koroglu M, Covic A. Erythropoiesis stimulatory agent- resistant anemia in dialysis patients: Review of causes and management. Blood Purif. 2010; 29:1-12.

58. Nibali L, Darbar U, Rakmanee T, Donos N. Anemia of inflammation associated with periodontitis: Analysis of two clinical studies. J Periodontol. 2019; 90:1252-1259.

59. World Health Organization, Report of WHO/UNICEF. Indicators and strategies for iron deficiency and anemia programmes. Geneva; 1994.

60. Hajishengallis G. Periodontitis: From microbial immune subversion to systemic inflammation. Nat Rev Immunol. 2015; 15:30-44.

6I. Koren O, Spor A, Felin J, Fåk F, Stombaugh J, Tremaroli V, et al. Human oral, gut, and plaque microbiota in patients with atherosclerosis. Proc Natl Acad Sci U S A. 20I I; 108:4592-4598.

62. Haraszthy VI, Zambon Jj, Trevisan M, Zeid M, Genco RJ. Identification of periodontal pathogens in atheromatous plaques. J Periodontol. 2000; 71:1554-1560.

63. Balejo RDP, Cortelli JR, Costa FO, Cyrino RM, Aquino DR, Cogo-Müller K, et al. Effects of chlorhexidine preprocedural rinse on bacteremia in periodontal patients: A randomized clinical trial. J Appl Oral Sci. 2017; 25:586-595.

64. Dagasso G, Conley J, Parfitt E, Pasquill K, Steele L, Laupland K. Risk factors associated with bloodstream infections in end-stage renal disease patients: a population-based study. Infect Dis (Auckl). 20 I8; 50:831-836.

65. Adeniyi $A B, N e l$ JD. An insight into renal disease associated with infective endocarditis. SA Heart. 2017; 4:24-29.

66. Mang-de la Rosa MR, Castellanos-Cosano L, Romero-Perez MJ, Cutando A. The bacteremia of dental origin and its implications in the appearance of bacterial endocarditis. Med Oral Patol Oral Cir Bucal. 2014; 19:e67. DOI: 10.4317/medoral. 19562.

67. Lockhart PB, Brennan MT, Thornhill M, Michalowicz BS, Noll J, Bahrani-Mougeot FK, et al. Poor oral hygiene as a risk factor for infective endocarditis-related bacteremia. J Am Dent Assoc. 2009; 140:1238-1244. 
68. Netzer ROM, Zollinger E, Seiler C, Cerny A. Infective endocarditis: Clinical spectrum, presentation and outcome. An analysis of 212 cases 1980-1995. Heart. 2000; 84:25-30.

69. Said S, Hernandez GT. The link between chronic kidney disease and cardiovascular disease. J Nephropathol. 2014; 3:99-104.

70. Higashi Y, Goto C, Jitsuiki D, Umemura T, Nishioka K, Hidaka T, et al. Periodontal infection is associated with endothelial dysfunction in healthy subjects and hypertensive patients. Hypertension. 2008; 51:446-453.

7I. Moura MF, Navarro TP, Silva TA, Cota LOM, Soares Dutra Oliveira AM, Costa FO. Periodontitis and endothelial dysfunction: Periodontal clinical parameters and levels of salivary markers interleukin- $\mid \mathbb{Q}$, tumor necrosis factor- $\bigotimes$, matrix metalloproteinase-2, tissue inhibitor of metalloproteinases-2 complex, and nitric oxide. J Periodontol. 2017; 88:778-787.

72. Orlandi M, Suvan J, Petrie A, Donos N, Masi S, Hingorani A, et al. Association between periodontal disease and its treatment, flow-mediated dilatation and carotid intima-media thickness: A systematic review and meta-analysis. Atherosclerosis. 2014; 236:39-46.

73. Jazani N, Savoj J, Lustgarten M, Lau W, Vaziri N. Impact of gut dysbiosis on neurohormonal pathways in chronic kidney disease. Diseases. 2019; 7:21. DOI:10.3390/diseases7010021.

74. Olsen I, Yamazaki K. Can oral bacteria affect the microbiome of the gut? J Oral Microbiol. 20 I9; I I: I 586422. DOI: I 0. I 080/20002297.20 19.1586422.

75. Walker MY, Pratap S, Southerland JH, Farmer-Dixon CM, Lakshmyya K, Gangula PR. Role of oral and gut microbiome in nitric oxidemediated colon motility. Nitric Oxide. 2018; 73:81-88.

76. Sato K, Takahashi N, Kato T, Matsuda Y, Yokoji M, Yamada M, et al. Aggravation of collagen-induced arthritis by orally administered Porphyromonas gingivalis through modulation of the gut microbiota and gut immune system. Sci Rep. 2017; 7(1):6955. DOl: I0.1038/ s41598-017-07196-7

77. Seedorf H, Griffin NW, Ridaura VK, Reyes A, Cheng J, Rey FE, et al. Bacteria from diverse habitats colonize and compete in the mouse gut. Cell. 20।4; I59:253-266.

78. Kumar M, Ji B, Babaei P, Das P, Lappa D, Ramakrishnan G, et al. Gut microbiota dysbiosis is associated with malnutrition and reduced plasma amino acid levels: Lessons from genome-scale metabolic modeling. Metab Eng. 2018; 49:128-142.

79. Hopper L, Cole M. Risk factors affecting nutritional status of dialysis patients: a quality improvement project. Nephrol News Issues. 2008; 22:26-7, 30- I, 34.

80. Lourenço TGB, Spencer SJ, Alm EJ, Colombo APV. Defining the gut microbiota in individuals with periodontal diseases: an exploratory study. J Oral Microbiol. 20 I 8; I0. DOI: I0.1080/20002297. 2018.1487741 .

81. Rodriguez Herrero E, Boon N, Pauwels M, Bernaerts K, Slomka V, Quirynen $M$, et al. Necrotrophic growth of periodontopathogens is a novel virulence factor in oral biofilms. Sci Rep. 2017; 7:1 107. DOl: 10.1038/s41598-017-01239-9.

82. Marcenes W, Kassebaum NJ, Bernabé E, Flaxman A, Naghavi M, Lopez A, et al. Global burden of oral conditions in 1990-2010. J Dent Res. 2013; 92:592-597.

83. Costantinides F, Castronovo G, Vettori E, Frattini C, Artero ML, Bevilacqua L, et al. Dental care for patients with end-stage renal disease and undergoing hemodialysis. Int J Dent. 2018; 2018: 9610892. DOI:10.1155/2018/9610892.

84. Abed $\mathrm{H}$, Burke M, Shaheen $\mathrm{F}$. The integrated care pathway of nephrology and dental teams to manage complex renal and postkidney transplant patients in dentistry: A holistic approach. Saudi J Kidney Dis Transpl. 20 I8; 29:766-774.
85. Kshirsagar A V., Craig RG, Moss KL, Beck JD, Offenbacher S, Kotanko P, et al. Periodontal disease adversely affects the survival of patients with end-stage renal disease. Kidney Int. 2009; 75:746-75I.

86. Bots CP, Brand HS, Poorterman JHG, Van Amerongen BM, Valentijn-Benz $\mathrm{M}$, Veerman $\mathrm{ECl}$, et al. Oral and salivary changes in patients with end stage renal disease (ESRD): A two year follow-up study. Br Dent J. 2007; 202: I-5.

87. Seymour RA, Thomason JM, Nolan A. Oral lesions in organ transplant patients. J Oral Pathol Med. 1997; 26:297-304.

88. Al-Mohaya M, Darwazeh, AMG, Bin-Salih S A-KW. Oral lesions in Saudi renal transplant patients. Saudi J Kidney Dis Transpl. 2009; 20:20-29.

89. Craig RG. Interactions between chronic renal disease and periodontal disease. Oral Dis; 2008; 14:1-7.

90. Zwiech R, Bruzda-Zwiech A. Does oral health contribute to post-Transplant complications in kidney allograft recipients? Acta Odontol Scand. 2013; 71:756-763.

91. Nylund KM, Meurman JH, Heikkinen AM, Furuholm JO, Ortiz F, Ruokonen HM. Oral health in patients with renal disease: a longitudinal study from predialysis to kidney transplantation. Clin Oral Investig. 20I 8; 22: 339-347. 